\title{
Fixed point for $F_{\perp}$-weak contraction
}

\section{Neeraj Garakoti*, Mahesh Chandra Joshi, Rohit Kumar}

\begin{abstract}
In this paper, we establish some fixed point results for $F_{\perp}$ weak contraction in orthogonal metric space and we give an application for the solution of second order differential equation.
\end{abstract}

\section{INTRODUCTION}

In 2017, Gordeji et al. [3] introduced the idea of orthogonal metric spaces and gave the following definitions.

Definition 1 ([3]). Let $X$ be a non-empty set and $\perp \subseteq X \times X$ be a binary relation. If $\perp$ satisfies the condition

there exists $x_{0} ;\left(\left(\right.\right.$ for all $\left.y ; y \perp x_{0}\right)$ or (for all $\left.\left.y ; x_{0} \perp y\right)\right)$,

then $(X, \perp)$ is called an orthogonal set (briefly $O$-set).

Example 1 ([3]). Let $X$ be the collection of people in world. Define orthogonality as $x \perp y$ if $x$ can donate blood to $y$. As we know blood group $A B^{+}$is universal acceptor and $O^{-}$is universal donor so $X$ is an $O$-set where orthogonal element is not unique.

\begin{tabular}{|c|c|c|}
\hline Type & You can give blood to & You can recive blood to \\
\hline$A^{+}$ & $A^{+}, A B^{+}$ & $A^{+}, A^{-}, O^{+}, O^{-}$ \\
$O^{+}$ & $O^{+}, A^{+}, B^{+}, A B^{+}$ & $O^{+}, O^{-}$ \\
$B^{+}$ & $B^{+}, A B^{+}$ & $B^{+}, B^{-}, O^{+}, O^{-}$ \\
$A B^{+}$ & $A B^{+}$ & Everyone \\
$A^{-}$ & $A^{-}, A^{+}, A B^{+}, A B^{-}$ & $A^{-}, O^{-}$ \\
$O^{-}$ & Everyone & $O^{-}$ \\
$B^{-}$ & $B^{-}, B^{+}, A B^{+}, A B^{-}$ & $B^{-}, O^{-}$ \\
$A B^{-}$ & $A B^{-}, A B^{+}$ & $A B^{-}, B^{-}, O^{-}, A^{-}$ \\
\hline
\end{tabular}

2020 Mathematics Subject Classification. Primary: 47H10; Secondary: 54H25. tion.

Key words and phrases. Orthogonal metric space, $F_{\perp}$-contraction, $F_{\perp}$-weak contrac-

Full paper. Received 2 June 2020, accepted 19 February 2021, available online 16 March 2021.

Author thanks UGC, New Delhi, for support under NET-JRF. 
Definition 2 ([3]). A sequence $\left\{x_{n}\right\}_{n \in \mathbb{N}}$ in $O$-set is called orthogonal sequence (briefly $O$-sequence) if either

$$
x_{n} \perp x_{n+1} \text { or } x_{n+1} \perp x_{n} \text {, for all } n \in \mathbb{N} .
$$

Definition $3([3])$. Let $(X, d, \perp)$ is said to be an orthogonal metric space if $(X, \perp)$ is an $O$-set and $(X, d)$ is a metric space. The space $X$ is orthogonally complete (briefly $O$-complete) if every Cauchy $O$-sequence is convergent.

Definition 4 ([3]). Let $(X, d, \perp)$ be an orthogonal metric space.

(1) A mapping $f: X \rightarrow X$ is said to be orthogonal contraction ( $\perp$ contraction) if

$$
d(f x, f y) \leq \lambda d(x, y) \text {, for all } x \perp y \text { and } 0<\lambda<1 .
$$

(2) A mapping $f: X \rightarrow X$ is called orthogonal preserving ( $\perp$-preserving) mapping if $x \perp y$ then $f(x) \perp f(y)$, and $f$ is said to be $\perp$-weakly preserving if $f(x) \perp f(y)$ or $f(y) \perp f(x)$ whenever $x \perp y$.

(3) A mapping $f: X \rightarrow X$ is orthogonal continuous ( $\perp$-continuous) if for each $O$-sequence $\left\{a_{n}\right\}_{n \in \mathbb{N}}$ in $X$ whenever $a_{n} \rightarrow a$ implies $f\left(a_{n}\right) \rightarrow$ $f(a)$ as $n \rightarrow \infty$. Also, $f$ is $\perp$-continuous if $f$ is $\perp$-continuous for each $a \in X$.

Generalizing Banach contraction, in 2012, Wardowski [8] introduced a new concept of contraction called $F$-contraction. Thereafter, $F$-contraction has been extended in different settings and various spaces with their applications, see $[1,2,4,7]$.

Definition 5 ([8]). Let $\mathcal{F}$ be the family of functions $F: \mathbb{R}_{+} \rightarrow \mathbb{R}$ such that

(F1) $F$ is strictly increasing, i.e. for all $\alpha, \beta \in \mathbb{R}^{+}$such that $\alpha<\beta \Longrightarrow$ $F(\alpha)<F(\beta)$;

(F2) For each sequence $\left\{\alpha_{n}\right\}_{n \in \mathbb{N}}$ of positive numbers $\lim _{n \rightarrow \infty} \alpha_{n}=0$ if and only if $\lim _{n \rightarrow \infty} F\left(\alpha_{n}\right)=-\infty$;

(F3) There exists $j \in(0,1)$ such that $\lim _{\alpha \rightarrow 0^{+}} \alpha^{j} F(\alpha)=0$.

A mapping $T: X \rightarrow X$ is said to be an $F$ - contraction if there exists $\tau>0$ such that, for all $x, y \in X$,

$$
d(T x, T y)>0 \Longrightarrow \tau+F(d(T x, T y)) \leq F(d(x, y)) .
$$

Example $2([8])$. Let $F: \mathbb{R}_{+} \rightarrow \mathbb{R}$ be given by a formula $F(\alpha)=\ln (\alpha)$. It is clear that $F$ satisfies $(F 1-F 3)$ for any $j \in(0,1)$. Each mapping $T: X \rightarrow X$ satisfying condition (1) is an $F$-contraction such that

$$
d(T x, T y) \leq e^{-\tau} d(x, y), \quad \text { for all } x, y \in X, T x \neq T y .
$$

It is clear that for $x, y \in X$ such that $T x=T y$, the inequalities $d(T x, T y) \leq$ $e^{-\tau} d(x, y)$ also holds, i.e. $T$ is Banach contraction.

Using the concept of $F$-contraction, Wardowski [8] generalized the Banach Contraction Principle and proved that every $F$-contraction in a complete metric space has a unique fixed point. Later in 2014, Wardowski [9] 
introduced the concept of $F$-weak contraction and established some fixed point results.

Recently, Sawangsup et al. [6] generalized $F$-contraction in orthogonal metric space and proved some fixed point results for $F_{\perp}$-contraction mappings.

Definition 6 ([6]). A map $T: X \rightarrow X$ is said to be orthogonal $F$ - contraction or $F_{\perp}$-contraction on an $O$-metric space $(X, \perp, d)$ if there exist $F \in \mathcal{F}$ and $\tau>0$ such that, for all $x, y \in X$ with $x \perp y$,

$$
d(T x, T y)>0 \Longrightarrow \tau+F(d(T x, T y)) \leq F(d(x, y)) .
$$

\section{Main Results}

Now, we generalize the condition of Wardowski [9] and prove some results in orthogonal metric spaces.

Definition 7. A map $T: X \rightarrow X$ is said to be orthogonal $F$-weak contraction on an $O$-metric space $(X, \perp, d)$ if there exist $F \in \mathcal{F}$ and $\tau>0$ such that, for all $x, y \in X$ with $x \perp y$,

$$
d(T x, T y)>0 \Longrightarrow \tau+F(d(T x, T y)) \leq F(M(x, y)),
$$

where

$$
M(x, y)=\max \left\{d(x, y), d(x, T x), d(y, T y), \frac{d(x, T y)+d(y, T x)}{2}\right\} .
$$

Theorem 1. Let $(X, \perp, d)$ be an $O$-complete metric space and $T$ be $a \perp$ preserving, $F_{\perp}$-weak contraction, $\perp$-continuous on $X$. Then, $T$ has unique fixed point in $X$.

Proof. From the definition of orthogonality, it follows that $x_{0} \perp f\left(x_{0}\right)$ or $f\left(x_{0}\right) \perp x_{0}$. Define a sequence $x_{n+1}=T x_{n}$, for all $n \in \mathbb{N}_{0}\left(\mathbb{N}_{0}=\mathbb{N} \cup\{0\}\right)$. If there exist $m \in \mathbb{N}_{0}$ such that $T x_{m+1}=T x_{m}$, then $x_{m}$ is fixed point of $T$. Thus we assume that $x_{n+1} \neq x_{n}$ for all $n \in \mathbb{N}_{0}$.

Since, $T$ is $\perp$-preseving, we have

$$
\text { either } x_{n} \perp x_{n+1} \text { or } x_{n+1} \perp x_{n} \text {, }
$$

for all $n \in \mathbb{N}_{0}$. This implies that $\left\{x_{n}\right\}$ is an $O$-sequence. By equation (3), we have

$$
\begin{aligned}
d\left(x_{n}, x_{n+1}\right)= & d\left(T x_{n-1}, T x_{n}\right)>0 \Longrightarrow \\
& \tau+F\left(d\left(T x_{n-1}, T x_{n}\right)\right) \leq F\left(M\left(x_{n-1}, x_{n}\right)\right),
\end{aligned}
$$

where

$$
M\left(x_{n-1}, x_{n}\right)=\max \left\{d\left(x_{n-1}, x_{n}\right), d\left(x_{n-1}, T x_{n-1}\right), d\left(x_{n}, T x_{n}\right),\right.
$$




$$
\begin{aligned}
& \left.\frac{d\left(x_{n}, T x_{n-1}\right)+d\left(x_{n-1}, T x_{n}\right)}{2}\right\} \\
= & \max \left\{d\left(x_{n-1}, x_{n}\right), d\left(x_{n}, x_{n+1}\right), \frac{d\left(x_{n-1}, x_{n+1}\right)}{2}\right\} \\
\leq & \max \left\{d\left(x_{n-1}, x_{n}\right), d\left(x_{n}, x_{n+1}\right), \frac{d\left(x_{n-1}, x_{n}\right)+d\left(x_{n}, x_{n+1}\right)}{2}\right\} \\
= & \max \left\{d\left(x_{n-1}, x_{n}\right), d\left(x_{n}, x_{n+1}\right)\right\} .
\end{aligned}
$$

If $\max \left\{d\left(x_{n-1}, x_{n}\right), d\left(x_{n}, x_{n+1}\right)\right\}=d\left(x_{n}, x_{n+1}\right)$, for some $n$, using equation (3), we have

$$
F\left(d\left(x_{n}, x_{n+1}\right)\right) \leq F\left(d\left(x_{n}, x_{n+1}\right)\right)-\tau<F\left(d\left(x_{n}, x_{n+1}\right)\right),
$$

which is a contradiction. Therefore, for all $n \in \mathbb{N}$, we have

$$
F\left(d\left(x_{n}, x_{n+1}\right)\right) \leq F\left(d\left(x_{n-1}, x_{n}\right)\right)-\tau .
$$

Hence, we get

$$
F\left(d\left(x_{n}, x_{n+1}\right)\right) \leq F\left(d\left(x_{0}, x_{1}\right)\right)-n \tau, \quad \text { for all } n \in \mathbb{N} .
$$

Taking limit as $n \rightarrow \infty$, we get

$$
\lim _{n \rightarrow \infty} F\left(d\left(x_{n}, x_{n+1}\right)\right)=-\infty .
$$

Hence, by the $(F 2)$ property

$$
\lim _{n \rightarrow \infty} d\left(x_{n}, x_{n+1}\right)=0 .
$$

By property $(F 3)$, there exist $j \in(0,1)$ such that

$$
\lim _{n \rightarrow \infty}\left(d\left(x_{n}, x_{n+1}\right)\right)^{j} F\left(d\left(x_{n}, x_{n+1}\right)\right)=0 .
$$

Multiplying (4) by $d\left(x_{n}, x_{n+1}\right)^{j}$, we get

$$
\left.\left(d\left(x_{n}, x_{n+1}\right)\right)^{j}\left(F\left(d\left(x_{n}, x_{n+1}\right)\right)-F d\left(x_{0}, x_{1}\right)\right)\right) \leq-n \tau\left(d\left(x_{n}, x_{n+1}\right)\right)^{j} \leq 0 .
$$

Taking limit and using equations (5) and (6), we get

$$
\lim _{n \rightarrow \infty}\left(n\left(d\left(x_{n}, x_{n+1}\right)\right)^{j}\right)=0 .
$$

Then, there exists $n_{1} \in \mathbb{N}$ s.t. $n\left(d\left(x_{n}, x_{n+1}\right)\right)^{j} \leq 1$, for all $n \geq n_{1}$, we get

$$
d\left(x_{n}, x_{n+1}\right) \leq \frac{1}{n^{\frac{1}{j}}} .
$$

Using tiangular inequality and equation (7), for all $m, n \in \mathbb{N}$ s.t. $m>n \geq$ $n_{1}$, we get 


$$
\begin{aligned}
d\left(x_{n}, x_{m}\right) & \leq d\left(x_{n}, x_{n+1}\right)+d\left(x_{n+1}, x_{n+2}\right)+\cdots+d\left(x_{m-1}, x_{m}\right) \\
& \leq \sum_{i=n}^{\infty} d\left(x_{i}, x_{i+1}\right) \leq \sum_{i=n}^{\infty} \frac{1}{i^{\frac{1}{j}}} .
\end{aligned}
$$

The series $\sum_{i=n}^{\infty} \frac{1}{i^{\frac{1}{j}}}$ is convergent. It follows that $\left\{x_{n}\right\}$ is a Cauchy $O$ sequence in $X$. Since $X$ is $O$-complete, there exists $z \in X$ such that $x_{n} \rightarrow z$ as $n \rightarrow \infty$. Since $T$ is $\perp$-continuous, we have

$$
T z=T\left(\lim _{n \rightarrow \infty} x_{n}\right)=\lim _{n \rightarrow \infty} x_{n+1}=z .
$$

Hence, $z$ is a fixed point of $T$.

For the uniqueness of fixed point, if $w$ is another fixed point of $T$, then from equation (3), we get

$$
\begin{aligned}
\tau+F(d(w, z))= & \tau+F(d(T w, T z)) \\
\leq & F(\max \{d(w, z), d(w, T w), d(z, T z), \\
& \left.\left.\frac{d(w, T z)+d(z, T w)}{2}\right\}\right)-\tau \\
= & F(d(w, z)),
\end{aligned}
$$

a contradiction that $\tau \leq 0$. So, $d(w, z)=0$, i.e., $w=z$.

Example 3. Let $X=[0,1] \cap \mathbb{Q}$ and the metric is defined by $d(x, y)=|x-y|$. Define the binary relation $\perp$ on $X$ by $x \perp y$ if $x y=0$ or $x$. It is easy to see that $0 \perp y$ for all $y \in X$. Hence, $(X, \perp)$ is an $O$-set. As we know the set of $\mathbb{Q}$ with Euclidian metric is not a complete metric space. Hence, $X$ is not a complete metric space but it is $O$-complete.

Let $T$ be a map defined as

$$
T(x)= \begin{cases}\frac{x}{2}, & \text { if } x \in[0,1) \cap \mathbb{Q} \\ 0, & \text { if } x=1\end{cases}
$$

It is easy to see that $\mathrm{T}$ is $\perp$-continuous and $\perp$-preserving. But, $T$ is not $F_{\perp}$-contraction because for $x=1$ and $y=3 / 4$, where 1 is orthogonal for each $y \in[0,1] \cap \mathbb{Q}$,

$$
d(x, y)=d\left(1, \frac{3}{4}\right)=\frac{1}{4}<\frac{3}{8}=d\left(T 1, T \frac{3}{4}\right)=d(T x, T y) .
$$

Hence, by equation (2), we get $\tau<0$ which is contradiction.

Now, we prove that $T$ satisfies the condition of $F_{\perp}$-weak contraction. Since, 0 is orthgonal for each $y \in[0,1] \cap \mathbb{Q}$. Therefore, for $x=0$ and $y \in(0,1) \cap \mathbb{Q}$, 


$$
\begin{aligned}
d(T 0, T y) & =d\left(0, \frac{y}{2}\right)=\frac{y}{2}<y=d(0, y) \\
& =\max \left\{d(0, y), d(0, T 0), d(y, T y), \frac{d(0, T y)+d(y, T 0)}{2}\right\} .
\end{aligned}
$$

Also, 1 is orthogonal for each $y \in[0,1] \cap \mathbb{Q}$. For $x=1$ and $y \in(0,1) \cap \mathbb{Q}$

$$
\begin{aligned}
d(T 1, T y) & =d\left(0, \frac{y}{2}\right)=\frac{y}{2}<1=d(1, T 1) \\
& =\max \left\{d(1, y), d(1, T 1), d(y, T y), \frac{d(1, T y)+d(y, T 1)}{2}\right\} .
\end{aligned}
$$

Hence, $T$ is $F_{\perp}$-weak contraction for $F(x)=\ln x$ and $\tau=\ln 2$. By Theorem (1), $T$ has a unique fixed point, i.e., $x=0$.

Remark 1. We get the result of Sawangsup et al. [6] as following corollary.

Corollary 1. Let $(X, \perp, d)$ be an $O$-complete metric space and $T: X \rightarrow X$ is an $F_{\perp}$-contraction with $\perp$-preserving and $\perp$-continuous. Then $T$ has a unique fixed point $z \in X$.

Corollary 2. Let $(X, \perp, d)$ be an $O$-complete metric space and $T: X \rightarrow X$. If there exist $F \in \mathcal{F}$ and $\tau>0$ such that, $T$ is $\perp$-continuous, $\perp$-preserving and $T$ satisfies

$$
\begin{aligned}
d(T x, T y)>0 \Rightarrow & \tau+F(d(T x, T y)) \\
\leq & F(\alpha d(x, y)+\beta d(x, T x)+\gamma d(y, T y)+ \\
& \delta[d(x, T y)+d(y, T x)])
\end{aligned}
$$

for all $x, y \in X$ with $x \perp y$, where $\alpha, \beta, \gamma, \delta \geq 0$ and $\alpha+\beta+\gamma+2 \delta<1$. Then $T$ has a unique fixed point $z \in X$.

Proof. For all $x, y \in X$, with $x \perp y$, we have

$$
\begin{aligned}
& \alpha d(x, y)+\beta d(x, T x)+\gamma d(y, T y)+\delta[d(x, T y)+d(y, T x)] \\
\leq & (\alpha+\beta+\gamma+2 \delta) \max \left\{d(x, y), d(x, T x), d(y, T y), \frac{d(x, T y)+d(y, T x)}{2}\right\} \\
\leq & \max \left\{d(x, y), d(x, T x), d(y, T y), \frac{d(x, T y)+d(y, T x)}{2}\right\} .
\end{aligned}
$$

Following the proof of theorem (1) we get the required result.

The following result is generalization of the result given in [5] where the existence of the fixed point for the mappings satisfying a contraction condition for the points of a closed ball was considered. 
Theorem 2. Let $(X, \perp, d)$ be an $O$-complete metric space and $T: X \rightarrow X$. If $T$ is $F_{\perp}$-weak contraction for all $x, y \in \overline{B\left(x_{0}, r\right)}, \perp$ - preserving and $\perp$ continuous on $X$. Moreover, for any $r>0$,

$$
\sum_{j=0}^{\mathbb{N}} d\left(x_{j}, T x_{j}\right) \leq r, \text { for all } j \in \mathbb{N} .
$$

Then there exists a point $z \in \overline{B\left(x_{0}, r\right)}$ s.t. $T z=z$.

Proof. We construct a sequence $x_{n}$ such that $x_{n}=T x_{n-1}$ and show that it is $O$-Cauchy sequence. First we claim that $x_{n} \in \overline{B\left(x_{0}, r\right)}$ for all $n$. We have,

$$
d\left(x_{0}, x_{1}\right)=d\left(x_{0}, T x_{0}\right) \leq r .
$$

Suppose, $x_{2}, x_{3}, \ldots, x_{n} \in \overline{B\left(x_{0}, r\right)}$. Following the proof of Theorem (1), we have

$$
F\left(d\left(x_{n}, x_{n+1}\right)\right) \leq F\left(d\left(x_{n-1}, x_{n}\right)\right)-\tau .
$$

Hence, by property $(F 1), d\left(x_{n+1}, x_{n}\right)<d\left(x_{n-1}, x_{n}\right)$. Thus,

$$
\begin{aligned}
d\left(x_{0}, x_{n+1}\right) & \leq d\left(x_{0}, x_{1}\right)+d\left(x_{1}, x_{2}\right)+\cdots+d\left(x_{n}, x_{n+1}\right) \\
& =\sum_{j=0}^{\mathbb{N}} d\left(x_{j}, T x_{j}\right) \leq r \Longrightarrow x_{n+1} \in \overline{B\left(x_{0}, r\right)} .
\end{aligned}
$$

Hence, $x_{n} \in \overline{B\left(x_{0}, r\right)}$, for all $n \in \mathbb{N}$. Again, following the proof of theorem (1), sequence $\left\{x_{n}\right\}$ is $O$-Cauchy sequence and $\left\{x_{n}\right\} \rightarrow z \in \overline{B\left(x_{0}, r\right)}$. Since $T$ is $\perp$-continuous, we have $T z=z$.

Remark 2. Taking $(X, d)$ a complete metric space in Theorem 2, we get the following result.

Corollary 3. Let $(X, d)$ be a complete metric space, $T: X \rightarrow X$ be a mapping, $r>0$ and $x_{0}$ be an arbitrary point in $X$. Suppose there exists $k \in[0,1)$ with

$$
d(T x, T y) \leq k d(x, y), \quad \text { for all } x, y \in \overline{B\left(x_{0}, r\right)},
$$

and $d\left(x_{0}, T x_{0}\right)<(1-k) r$. Then there exists a unique point $z \in \overline{B\left(x_{0}, r\right)}$ such that $z=T z$.

\section{Applications}

Now, we apply our result to prove the existence theorem for the solution of the second order differential equation. which represents motion of spring under exterior force (for more details see [7]) and given by;

$$
\left\{\begin{array}{l}
\frac{\mathrm{d}^{2} u}{\mathrm{~d} t^{2}}+\frac{c}{m} \frac{\mathrm{d} u}{\mathrm{~d} t}=K(t,(u(t)) \\
u(0)=0, \quad u^{\prime}(0)=a
\end{array}\right.
$$


where $K:[0, I] \times \mathbb{R}^{+} \rightarrow \mathbb{R}$ is a continuous function and $I>0$.

Above problem is equivalent to the integral equation

$$
u(t)=\int_{0}^{t} G(t, s) K(s, u(s)) \mathrm{d} s, \quad t \in[0, I],
$$

where $G(t, s)$ is the Green's function, given by

$$
G(t, s)=\left\{\begin{array}{cc}
(t-s) e^{\tau}(t-s), & 0 \leq s \leq t \leq I \\
0, & 0 \leq t \leq s \leq I
\end{array}\right.
$$

where $\tau>0$ is a constant, calculated in terms of $c$ and $m$.

Let $X=C\left([0, I], \mathbb{R}^{+}\right)$be the set of all non negative continuous real functions defined on $[0, I]$ with

$$
u \perp v \quad \Longleftrightarrow \quad u(t) v(t) \geq 0,
$$

for an arbitrary $u, v \in X$. We define

$$
\|u\|_{\tau}=\sup _{t \in[0, I]}\left\{|u(t)| e^{-2 \tau t}\right\}, \quad \text { where } \tau>0 .
$$

Hence, $(X, d)$ is an $O$-complete metric space. Consider the self-map $T$ : $X \rightarrow X$ defined as

$$
T u(t)=\int_{0}^{t} G(t, s) K(s, u(s)) \mathrm{d} s, \quad t \in[0, I] .
$$

Clearly, if equation (8), possess a solution then it must be fixed point of $T$.

Theorem 3. Suppose:

(i) $K$ is increasing function,

(ii) $u \perp v \Longleftrightarrow u(t) v(t) \geq 0$,

(iii) there exists $\tau>0$ such that

$$
|K(s, u)-K(s, v)| \leq \tau^{2} e^{-\tau} M(u, v),
$$

for all $s \in[0, I], u, v \in \mathbb{R}^{+}$, where

$$
M(u, v)=\max \left\{|u-v|,|u-T u|,|v-T v|, \frac{|u-T v|+|v-T u|}{2}\right\},
$$

such that

$$
M_{\tau}(u, v)=\sup _{t \in[0, I]}\left\{e^{-2 \tau t} M(u, v)\right\} .
$$

Then the equation (9) has a unique solution.

Proof. Since $X=C\left([0, I], \mathbb{R}^{+}\right)$is an $O$-complete metric space. It is clear that $T$ is $\perp$-continuous and $\perp$-preserving.

Now, for all $u, v \in X$ with $u \perp v$ and $T u(t) \neq T v(t)$, we have 


$$
\begin{aligned}
|T u(t)-T v(t)| & \leq \int_{0}^{t} G(t, s)|K(s, u(s))-K(s, v(s))| \mathrm{d} s \\
& \leq \int_{0}^{t} G(t, s) \tau^{2} e^{-\tau} M(u, v) \mathrm{d} s \\
& =\int_{0}^{t} G(t, s) \tau^{2} e^{-\tau} e^{2 \tau s} e^{-2 \tau s} M(u, v) \mathrm{d} s \\
& \leq \tau^{2} e^{-\tau} M_{\tau}(u, v) \int_{0}^{t} G(t, s) e^{2 \tau s} \mathrm{~d} s \\
& =\tau^{2} e^{-\tau} M_{\tau}(u, v) \int_{0}^{t} e^{2 \tau s}(t-s) e^{\tau(t-s)} \mathrm{d} s \\
& =\tau^{2} e^{-\tau} M_{\tau}(u, v) e^{\tau t} \int_{0}^{t} e^{\tau s}(t-s) \mathrm{d} s \\
& =\tau^{2} e^{-\tau} M_{\tau}(u, v) e^{\tau t} \times \frac{e^{\tau t}}{\tau^{2}}\left(1-\tau t e^{-\tau t}-e^{-\tau t}\right) \\
& \leq e^{-\tau} M_{\tau}(u, v) e^{2 \tau t} ; \quad\left(1-\tau t e^{-\tau t}-e^{-\tau t}\right) \leq 1 .
\end{aligned}
$$

Therfore,

$$
\begin{aligned}
|T u(t)-T v(t)| e^{-2 \tau t} & \leq e^{-\tau} M_{\tau}(u, v) \\
\|T u-T v\|_{\tau} & \leq e^{-\tau} M_{\tau}(u, v)
\end{aligned}
$$

Taking logarithms, we get

$$
\begin{aligned}
\ln \left(\|T u-T v\|_{\tau}\right) & \leq \ln \left(e^{-\tau} M_{\tau}(u, v),\right. \\
\tau+\ln \left(\|T u-T v\|_{\tau}\right) & \leq \ln M_{\tau}(u, v) .
\end{aligned}
$$

$T$ satisfies all the criteria for $F(x)=\ln (x)$ of Theorem 1 . Hence, we get a unique solution of equation (9).

\section{REFERENCES}

[1] H. Ayadi, E. Karapinar and H. Yazidi, Modified F- contaction via $\alpha$-admissible mappings and application to integral equation, Filomat, 5 (2017), 1141-1148.

[2] H. Baghani, M.E. Gordji and M. Rameani, Orthogonal sets: The axiom of choice and proof of a fixed point theorem, Journal of Fixed Point Theory and Applications, 18 (2016), 465-477.

[3] M.E. Gordji, M. Rameani, M. De La Sen, and Y.J. Cho, On orthogonal sets and Banach fixed point theorem, Fixed Point Theory, 18 (2017), 569-578.

[4] A. Hussain and M. Arshad, New type of multivalued F-Contraction involving Fixed Point on Closed Ball, Journal of Mathematics and Computer Science (JMCS), 17 (2) (2017), 246-254.

[5] E. Kryeszig, Introductory functional analysis with applications, John Wiley \& Sons, New York, 1989. 
[6] K. Sawangsup, W. Sintunavarat and Y.J. Cho, Fixed point theorems for orthogonal $F$-contraction mappings on O-complete metric space, Journal of Fixed Point Theory and Applications, 22 (2020).

[7] D. Singh, V. Joshi, M. Imdad and P. Kumam, Fixed point theorems via generalized F-contractions with applications to functional equations occurring in dynamic programming, Journal of Fixed Point Theory and Applications, 19 (2017), 1453-1479.

[8] D. Wardowski, Fixed point theory of a new type of contractive mappings in complete metric spaces, Fixed Point Theory and Applications, 2012 (2012), Article ID: 94, 6 pages.

[9] D. Wardowski and N. Van Dung, Fixed point of F-weak contractions on complete metric spaces, Demonstratio Mathematica, 47 (2014), 146-155.

\section{Neeraj Garakoti}

Department of Mathematics

D.S.B. Campus, Kumaun University

NAINITAL-263002

INDIA

E-mail address: neerajgarakoti@gmail.com

Mahesh Chandra Joshi

Department of Mathematics

D.S.B. Campus, Kumaun University

NAINITAL-263002,

INDIA

E-mail address: mcjoshi69@gmail.com

\section{Rohit Kumar}

Department of Mathematics

D.S.B. Campus, Kumaun University

NAINITAL-263002

INDIA

E-mail address: rohitk0351@gmail.com 